Cinémas

Revue d'études cinématographiques

Journal of Film Studies

\title{
India Song ou le temps tragique
}

\section{Sémir Badir}

Volume 5, numéro 1-2, automne 1994

Le Temps au cinéma

URI : https://id.erudit.org/iderudit/1001009ar

DOI : https://doi.org/10.7202/1001009ar

Aller au sommaire du numéro

Éditeur(s)

Cinémas

ISSN

1181-6945 (imprimé)

1705-6500 (numérique)

Découvrir la revue

Citer cet article

Badir, S. (1994). India Song ou le temps tragique. Cinémas, 5(1-2), 123-133.

https://doi.org/10.7202/1001009ar

\section{Résumé de l'article}

L'auteur démontre ici que le film India Song de Marguerite Duras (1975) constitue une résistance du lieu filmique, en ce sens qu'il révèle une indifférence à l'égard des présupposés dramatiques habituels : contexte réaliste, soin du vraisemblable et ordre spatio-chronologique. Cette résistance fait d'India Song une tragédie, un film tragique. d'utilisation que vous pouvez consulter en ligne.

https://apropos.erudit.org/fr/usagers/politique-dutilisation/ 


\title{
India Song ou le temps tragique
}

\section{Sémir Badir}

\begin{abstract}
RÉSUMÉ
L'auteur démontre ici que le film India Song de Marguerite Duras (1975) constitue une résistance du lieu filmique, en ce sens qu'il révèle une indifférence à l'égard des présupposés dramatiques habituels: contexte réaliste, soin du vraisemblable et ordre spatiochronologique. Cette résistance fait d'India Song une tragédie, un film tragique.
\end{abstract}

\section{ABSTRACT}

The author's purpose in this article is to demonstrate that Marguerite Duras's India Song (1975) constitutes a site of filmic resistance, in that it displays a lack of concern with ordinary dramatic presuppositions - the realist context, the regard for verisimilitude and spatiochronologic ordering. This resistance makes of India Song a tragedy, a tragic film.

Au cinéma, le régime habituel pour relater une histoire, c'est le drame. Le drame a un cousin, la comédie, dont le divertissement n'est possible que s'il se heurte à des obstacles dramatiques. Le drame connaît également des enfants terribles - Antonioni, Fellini, Godard - qui, à coup de négations et de mises en abyme, s'attaquent aux artifices dramatiques. Ces artifices, on dira en gros que le drame cinématographique les a empruntés, en les aménageant à sa matière, au romanesque. En affinant à peine, on reconnaît que le drame possède quelques caractéristiques constantes: un contexte, constitué par un temps et un lieu réalistes, soutenu par un souci du vraisemblable, et présenté dans une linéarité au moins préliminairement attendue. Il va de soi 


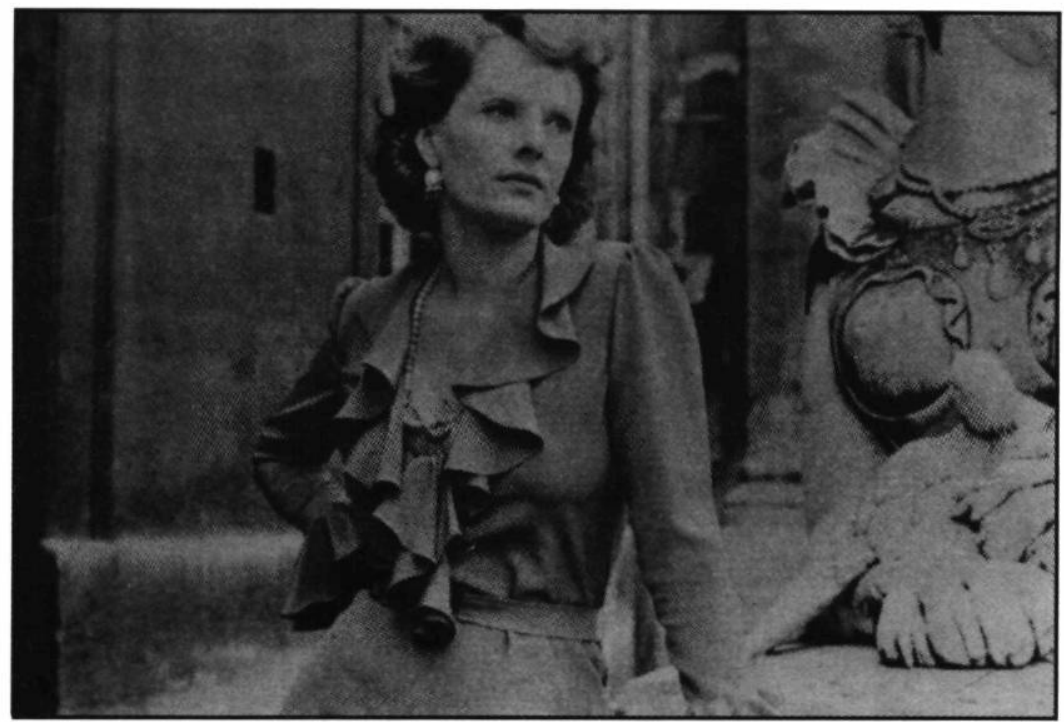

\section{India Song de Marguerite Duras (1975)}

Collection Cinémathèque québécoise

que chacune de ces caractéristiques a pu être joyeusement mise à l'envers, mais ce faisant, on n'a fait que renforcer leur institution: on s'inscrit dans un mode de représentation institutionnel quand bien même on cherche à le compromettre.

On imagine aisément ce que pourrait être un récit dramatique d'India Song. Rien de plus convenu en effet que le triangle amoureux, l'oisiveté dans les colonies, la folie de l'homme seul, la passion. Oui, même la passion est "convenable" pourvu qu'elle s'aligne sur un cliché ronéotypé d'un film à l'autre. Il suffirait de laisser voir un peu la mendiante, de faire bouger les lèvres des personnages, d'arrêter cette musique intempestive et d'évoquer quelques souvenirs en flash back à la place des plans d'extérieurs pour que se rétablisse heureusement la médiocrité du mélodrame.

L'accès au tragique, en revanche, nécessite l'abandon, ou plutôt l'indifférence absolue à l'égard des présupposés dramatiques. Rendre un sens tragique, c'est "oublier", sans nécessairement les rejeter in facto, le contexte réaliste, le soin du vraisemblable et l'ordre spatio-chronologique dans lequel le récit cinématographique se déploie d'ordinaire. 
Mais, la résistance de ce que nous allons appeler le lieu filmique va, en réalité, faire d'India Song une tragédie, un film tragique.

Au reste, avec cette assertion, je ne m'avance pas trop, car l'auteure était tout à fait consciente de la qualité de son film:

Ce qui peut être nommé tragique, ici, je crois que ce n'est pas la teneur de l'histoire racontée, ni le genre auquel elle se rapporte dans la classification habituelle, c'est aussi le contraire: c'est ce à partir de quoi cette histoire se raconte qui peut être dit tragique, c'est-àdire la mise en présence corrélative et de la destruction de cette histoire par la mort et l'oubli, et de cet amour cependant que détruite elle continue à prodiguer (Duras, p. 22).

Deleuze, Réda Bensmaia l'a rappelé lors d'une discussion que nous avons eue, parle de l'interstice inhérent à l'œuvre de Marguerite Duras comme d'un impossible, qui est à vrai dire un "incompossible", une impossibilité de tenir ensemble le passé et le présent, un amour et l'histoire de cet amour.

La même idée transparaît chez Hölderlin (vers 1800) :

La signification des tragédies s'explique le plus facilement par le paradoxe. Car toute chose originelle, du fait que tout bien est équitablement et également réparti, apparaît non pas dans sa force originelle, mais plutôt dans sa faiblesse, de sorte qu'au vrai, c'est bien à la faiblesse de chaque ensemble qu'appartient la lumière de la vie et l'apparition (p. 644).

Et chez Hegel (1837):

Ainsi "par action tragique" se trouve posée une contradiction non résolue qui, tout en devenant une réalité, ne peut cependant pas se maintenir dans le réel et s'y imposer comme un élément substantiel, mais n'est justifiée que pour autant qu'elle offre une possibilité de résorption (p. 250).

Enfin, dans les pas de la lecture nietzschéenne, Clément Rosset a précisé la condition du tragique dans le rapport particulier qu'il entretient avec le temps:

Lidée du tragique repose tout entière dans un rapport entre deux situations, elle est la représentation ulté- 
rieure du passage d'un état à un autre; en conséquence, on peut parler de mécanisme tragique, pas de situation tragique. Mais il faut préciser que le tragique n'apparaît que lorsque nous représentons - après coup - le mécanisme tragique. [...] Il y a donc deux idées essentielles et complémentaires dans le sentiment tragique: l'idée du mouvement et l'idée de l'immobilité soudain fondues en une seule intuition; l'idée d'un mécanisme figé, qu'on pourrait représenter sur un tableau, sans tenir compte du temps, car il s'agit d'un schéma tragique qui usurpe le temps, se sert du temps, fait comme sül était le temps (p. 7-8).

Incompossible dont on souffre le va-et-vient, contradiction non résolue cependant que réalisée, paradoxe, tel est bien, me semble-t-il, le motif du procès dont India Song est le lieu de résorption. Qu'on me permette d'évoquer rapidement les aspects majeurs de ce procès autour de quatre éléments fondamentaux du cinéma: dans la bande sonore, les voix et la musique dans la bande-image, les acteurs et le décor.

Les voix, donc, pour commencer. Elles se regroupent en deux familles. Les premières sont extra-diégétiques: elles entreprennent un commentaire des images et construisent petit à petit, en s'aidant les unes les autres à raviver leur mémoire, l'histoire d'amour entre le vice-consul de Lahore et Anne-Marie Stretter. Elles dialoguent sur le mode du présent historique. Ce mode est celui de la mémoire encore en train de s'établir, il rend compte de deux temps - le présent de la narration et le passé de l'histoire racontée - et amorce par là-même leur rencontre, voire leur fusion. De manière à ressusciter l'histoire du vice-consul et d'Anne-Marie Stretter :

$$
\begin{aligned}
& \text { V2.: Le soir, ils dansaient? } \\
& \text { V1.: Ils dansent. }
\end{aligned}
$$

en même temps que cet amour raconté atteint, par imprégnation, les voix qui l'énoncent:

$$
\begin{aligned}
& \text { V1.: De quoi avez-vous peur? } \\
& \text { V2. [bas et tendu] : Anne-Marie Stretter. } \\
& \text { [...] }
\end{aligned}
$$


V1.: Sur quoi pleurez-vous?

[silence]

V2. : Je vous aime jusqu'à ne plus voir, ne plus enten-

dre, mourir...

Les voix de la seconde famille appartiennent manifestement aux personnages, mais ne sont néanmoins jamais montrées dans le profilmique visuel. C’est le procédé que Marguerite Duras appelle "les bouches closes»:

Il ne fallait à aucun moment "faire revivre" cette histoire et entraîner, ne fût-ce que pendant quelques minutes, une identification entre ces gens de l'écran, ces acteurs, et ces autres gens de Calcutta. Il fallait que ce qui avait été supposé avoir été dit par ces gens, dans ces conversations, soit redit, mais en présence du signe concret de leur mort - de même que ce qui était $v u$ l'était en présence de la photographie de la morte. Et j'ai trouvé les bouches closes: tandis qu'ils parlent, leurs bouches se taisent (Duras, p. 21-22).

Ces voix-là participent donc également à l'effort de reconstruction mémorielle du film: elles sont tirées de l'oubli, mais leur exhortation ne fait aucunement illusion: elles sont là pour mémoire. Donc, pas d'identification. Néanmoins, ce qui a subsisté, c'est le désir d'identification, l'implication volontaire des acteurs dans l'histoire. C'est la prise en charge - même résistante - , par l'acteur en tant qu'acteur, des sentiments, des émotions, des actes, bref, de la vie de son personnage. Cette vie ne sera pas considérée par l'acteur comme fictive mais comme passée. Il ne s'agit pas dès lors pour lui de faire "revivre" ce passé (ce qui serait une position fictive) mais de lui rendre accès au présent par sa voix et par son corps.

D'ailleurs, dans le film, ce désir d'identification est renforcé par des effets de réalité: les voix d'ambiance, les bruits des oiseaux et de la mer, le rapprochement qu'il est permis de faire entre la voix et le corps de l'acteur, prêtés tous deux au même personnage (alors qu'on aurait pu imaginer par exemple que ce serait la voix de Viviane Forrester qui viendrait suppléer celle d'Anne-Marie Stretter pendant que Delphine Seyrig apparaît à l'écran). C'est donc qu'à l'intérieur du film, il y a deux mouve- 
ments contradictoires; l'un expose l'artefact tandis que l'autre, tout en l'admettant, propose de le nier par des effets de réalité. Assurant une mise en abyme désabusive (ce que d'aucuns appellent la "déréalisation" d'India Song), le film engendre en même temps le désir de réduire l'écart entre la diégèse et le récit, non pas cependant par l'illusion traditionnelle de la représentation, mais bien par l'annihilation de la logique temporelle, par l'anéantissement du temps narratif dans le temps de l'énoncé.

De la musique, Marguerite Duras confiait à Nicole-Lise Bernheim qu'elle a "[...] une sorte de fonction impitoyable. Pendant la réception, à de rares exceptions près, elle revient toutes les minutes quel que soit l'événement. Elle vient malgré l'événement" (p. 121).

La bande musicale d'India Song est en effet, comme les voix, extra-diégétique. Il n'est jamais fait mention dans l'image que la musique y soit jouée, sauf peut-être lors de la vue d'un piano. Néanmoins, la musique n'est pas sans rapport avec l'image ni avec le reste de la bande-son. Ainsi, la variation de Beethoven est jouée lorsqu'on parle du passé vénitien d'Anne-Marie Stretter ou lorsqu'elle s'en va, à la fin du film, vers son suicide. Et la plupart des airs de danse sont joués pendant la réception. Il y a donc, dans ces rapprochements, une potentialité de représentation. La musique "aurait pu " être jouée là, si ce que l'on voyait à l'image était vraiment le passé recomposé - mais il ne l'est pas. Elle procède donc de cet effort de mémoire dont India Song est le lieu et l'objet. Mais justement, parce que cette fonction reste virtuelle, la musique n'a pas non plus de motivation propre dans la diégèse. Sa position est indéfinie. Dès lors, soit on devait la charger d'une signification en choisissant sa place (c'est ce qui se fait dans ce que Duras appelle "le cinéma qui triche»), soit elle impose elle-même sa présence, pour ne pas être - jamais dans l'embarras de cette présence.

La systématicité mécanique de la musique dans India song affirme l'extra-diégèse comme étant le réel du film. Elle dénonce le procédé d'enregistrement filmique, certes, mais elle le dénonce comme vrai (cinématographique) et non comme faux (réel).

La méthode de "jeu» employée dans India Song est devenue assez célèbre. Vous vous la rappelez peut-être, lors des répéti- 
tions et des prises de vue, les textes dits par les voix des quatre narrateurs, des invités de la réception et des acteurs, les textes décrivant les plans eux-mêmes étaient entendus par les acteurs (par exemple, si l'on disait qu' Anne-Marie Stretter entre dans le salon particulier, regarde le parc, Delphine Seyrig effectivement, au même moment, entrait, regardait dans le parc). Les acteurs avaient donc l'impression d'illustrer une scène déjà décrite, exprimée. Ils n'avaient plus qu'à la "représenter " à l'image, sans la faculté d'ajouter leur propre signification aux gestes qu'ils étaient en train d'exécuter.

Les acteurs sont embarrassés de leur corps parce que ceux-ci sont toujours trop présents et n'ont aucune chance de participer directement au travail de la mémoire (c'est pourquoi il faut les déguiser, leur imprimer une démarche, un comportement); les acteurs ne réagissent qu'avec une extrême réticence, une torpeur exténuante, comme s'ils avaient peur de mal faire, d'ajouter leur présent gros et gras et d'obstruer la voie aux temps virtuels désirés. Quant à leurs regards, ils sont lourds ou absents, toujours au point qu'ils se désarticulent des corps: ne fuient pas ni ne s'animent, mais se figent comme s'ils étaient hors du temps. Le temps lui-même est ainsi tout entier virtuel dans le regard des acteurs. L'image que produit India Song, pour reprendre un terme employé par Gilles Deleuze, cristallise sa propre image virtuelle, elle se constelle de toutes les virtualités du temps, passé et présent, diégétique et cinématographique, subjective et objective, visuelle et auditive, jusqu'à former une image-produit, coalescente, autour d'un point d'indiscernabilité.

Je vois [les comédiens], oui, absents, donc rendus à eux-mêmes, dans ce film, nettoyés de ce self-regard qui recouvre en général le jeu de la vedette et prive le spectateur de l'apercevoir de l'autre côté d'elle-même, de sa fonction. Je vois leur absence parallèle à la nôtre lorsque nous les regardons dans le film. Et qu'ainsi, égarés, nous nous rencontrons (Bernheim, p. 15).

Égarés, certes, nous pourrions l'être. Car, bien sûr, il n'y pas d'ambassade de France à Calcutta; le lieu, l'espace du film, est une pure invention. Pourtant, il n'était pas utile d'aller s'inspirer des décors "naturels" et "originaux" de l'Inde ni de les copier, 
pour la raison qu'il ne s'agit pas d'un film historique. Reste donc, pour mobiliser un lieu, soit la convention abstraite (la ruine Rothschild), soit l'illusion réaliste (l'hôtel Pommereux). Or, ce n'est ni pour l'un ni pour l'autre, mais pour l'un-etl'autre, et même pour l'un-dans-l'autre-et-réciproquement que Marguerite Duras va opter. Entre Rothschild et Pommereux se joue la suspension des lieux réels et fictifs et l'investigation du lieu filmique: vrai parce que vraiment faux, susceptible de réalité parce qu'au-delà de sa virtualité fictionnelle.

De ce fait, le lieu filmique se sépare de l'espace imaginaire qu'une diégèse lui assignerait d'ordinaire. Ces extérieurs d'hôtels s'inscrivent à la fois dans un présent fictif (ils représentent les bâtisses de la colonie aux Indes tels qu'ils sont aujourd'hui, décrépits) et dans le passé d'eux-mêmes (le vide de ces images nous amène à les considérer tels). Ainsi, les façades extérieures, lézardées, rendent-elles compte dans une pleine virtualité, en un souvenir pur, du passé de l'histoire présentée; elles nous le livrent en ce qu'il est inimaginable. Ces plans ne forment pas des images-souvenirs mais des images en souvenir, qui ne sont pas elles-mêmes des souvenirs, mais sont travaillées par la mémoire. À la question "Qu'est-ce qui s'est passé?", India Song ne donne à entendre que son doute: peut-être, cela aurait pu se passer comme cela.

Le passé et l'«autre réalité" ne préexistent plus à l'image, mais se déduisent d'elle. Dès lors, il n'y a plus de hors-champ, plus d'espace en dehors de l'image. L'image, se coupant du monde de la réalité, a renoncé à son extériorité: elle s'est rendue libre de ce qui dépendait d'elle. Cet anéantissement du hors-champ vraisemblable est également manifesté par la sorte de montage primitif de la deuxième partie où alternent des plans d'intérieurs cohérents diégétiquement (ils évoquent la réception) et des plans d'extérieurs extra-diégétiques homogènes (ils exposent des façades).

Le film, en se donnant d'une façon si tranchée un dedans et un dehors, annule par la même occasion la possibilité de leur connexion supposée. Car aucun lien logique ne peut être présumé entre ces deux séries d'images; ils sont dans un rapport d'exclusion l'un à l'autre. Comme un envers et un endroit. Le 
tout, dans ce film, ce n'est pas l'ouvert mais le dehors (selon la terminologie deleuzienne). Et l'essentiel se passe dans l'interstice de ces contraires: dans un "[...] espacement qui fait que chaque image s'arrache au vide et y retombe" (Deleuze, p. 234).

$\mathrm{Ou}$, pour en revenir à ce que nous disions du tragique, le dedans et le dehors sont, dans India Song, incompossibles. De même qu'elle évite à tout prix la confusion entre le passé et le présent, Duras ne peut pas supporter cet affreux mélange, ce mixage bassement complaisant, entre son histoire et le monde. Le monde ne peut pas être ramené au statut de "contexte", on ne peut réduire l'Autre à un prolongement de soi. C'est pourquoi il n'y a pas de hors-champ actualisable dans India Song: pas d'ouverture mais des espaces incompossibles. S'ils doivent exister, être rendus possibles, ce ne peut être que dans une dissociation radicale, dans une fuite éperdue, prismatique, de leur référence à une (soi-disant) commune réalité.

Le tragique ne procède pas par continuité mais par dissociation. Aussi a-t-on pu dire qu'il constitue un schéma posé contre le temps, suppléant le temps. Le "temps tragique", expression évidemment paradoxale, quasi oxymorique, connaît selon Clément Rosset deux caractéristiques. Premièrement, c'est un temps raide, hiératique, "déroulé comme un ressort". Je n'ai pas besoin d'insister longuement sur la pertinence de cet aspect dans India Song; c'est bien de la raideur que nous percevons immédiatement dans le jeu des acteurs et dans le défilement des plans d'extérieur (le soleil, le parc, le Gange). La musique, elle aussi, est conduite avec une fixité mécanique. Les voix même sont tendues, désincarnées. Cette rigidité à laquelle participent tous les éléments du film, c'est celle du temps filmique, qui est à la fois temps de la représentation et temps du récit, temps suspendu et abstrait entre ces deux nécessités, qui en accuse l'étrangeté. Tout le film baigne en effet dans une sorte de torpeur, d'indolence, de résignation. Le lien sensori-moteur entre les images y est distendu, pas au point de rompre, mais de façon à perdre taute impression de naturel: c'est un lien incoercible cependant que rapporté à un schéma immobile. Ceci nous amène à la seconde caractéristique du temps tragique qui est d'être un temps inversé. "Il ne se passe rien dans ce film", ai-je souvent entendu 
dire. C'est vrai : car tout déjà est passé. Le tragique est la représentation d'un inéluctable à la recherche de son origine. India Song tente ce parcours immobile, balisé par le travail de la mémoire. Et, dans une scène au moins, je vois le tragique se réaliser, le mécanisme tragique se répéter, l'incompossible avoir lieu : en dépit des acteurs, des voix, de la musique et du décor.

Cette scène, je l'appelle la scène du cri. Elle succède à la danse du couple fou: celle d'Anne-Marie Stretter et du vice-consul, alors qu'ils se sont déclaré un amour réciproque, au-delà de l'" histoire d'amour " qu'ils (ne) pourraient (pas) avoir ensemble; s'y mêle aussi leur souffrance commune de l'Inde.

VOIX DU VICE-CONSUL. Oui... Vous êtes avec moi devant Lahore. Je le sais... Vous êtes en moi... Je vous emmènerai en moi, et vous tirerez avec moi sur les lépreux de Shalimar, qu'y pouvez-vous? Je n'avais pas besoin de vous inviter à danser pour vous connaître. Et vous le savez.

VOIX D'ANNE-MARIE STRETTER. Je le sais.

VOIX DU VICE-CONSUL. Il est tout à fait inutile qu'on aille plus loin, vous et moi... Nous n'avons rien à nous dire... Nous sommes les mêmes...

VOIX D'ANNE-MARIE STRETTER. Je crois ce que vous venez de dire...

VOIX DU VICE-CONSUL. Les histoires d'amour, vous les vivez avec d'autres. Nous n'avons pas besoin de ça...

D'un commun accord, ils décident de provoquer un incident public: le vice-consul va hurler d'amour pour elle; il crie son nom de Venise dans Calcutta désert: Anna-Maria Guardi. La protestation est à la base de ce qui anime le comportement du vice-consul. Protester contre le Dedans de son amour pour Anne-Marie Stretter: il hurle. Alors, on le chasse, on le rejette Dehors - on voudrait que la tragédie n'ait rien à faire dans cette sombre histoire de drame.

Mais voilà que ce cri surprend le spectateur par sa singulière apparence: il est lointain, résonne dans les salons de l'ambassade, puis perd petit à petit de son volume, alors qu'on emmène le vice-consul. La baisse progressive de volume du cri suggère très fortement un statut de voix off (alors que les autres voix, 
qu'elles soient d'ambiance ou très claires ne connaissent pas de variations), et par conséquent s'ouvre la possibilité du horschamp. Voix off et hors-champ, deux choses dont nous semblions pourtant assurés qu'elles ne pouvaient avoir cours dans ce film, se manifestent dans le sursaut tragique du vice-consul. Comme si le cristal temporel trouvait là son point intersticiel, et que la vérité du tragique transcendait les limites de sa représentation.

Ce que produisent les décalages dans India Song, c'est une fuite absolue, la rencontre impossible entre une histoire qui n'a plus de lieu et des lieux qui n'ont pas d'histoire. Dans l'interstice même, c'est la fuite du temps qui court de l'un à l'autre, d'une virtualité à l'autre. D'une part, le bal originaire reste virtuel à cause des effets de réalité qui stoppent net tout élan vers l'illusion cinématographique, mais d'autre part, la représentation manquée de ce passé a perdu toute actualité en se heurtant au Dehors, incarné par le vice-consul (mais aussi par la mendiante, par l'océan, ces grands absents de la bande-image), qui crée une coupure irrationnelle entre les différentes scènes de la représentation, de sorte que cette dernière doit abandonner la linéarité spatio-temporelle qui aurait fondé une pleine actualité. C'est pourquoi India Song reste une fiction, et non un documentaire ni un "essai"; seulement, cette fiction est uniquement - c'est là sa beauté - un lieu filmique: au-delà du champ cinématographique conventionnel afin d'obtenir une nécessité originale à l'interstice de tout ce qui le compose (la diégèse, l'Histoire, l'autobiographie, le cinéma).

Université de Liège

\section{OUVRAGES CITÉS}

Bernheim, Nicole-Lise. Marguerite Duras tourne un film... Paris: Albatros, 1975.

Duras, Marguerite. “Notes sur India Song”, Marguerite Duras. Paris: Albatros, 1979.

Deleuze, Gilles. Cinéma 2. L'Image-temps. Paris: Minuit, 1985.

Hegel, G. W. T. Esthétique. Tome III. Paris: Aubier/Montaigne, 1944

Hölderlin, Friedrich. Euvres. Paris: Gallimard, 1967.

Rosset, Clément. La Philosophie tragique. Paris : P.U.F., 1991. 